\title{
Neue TARMED-Position Psychosomatische Gruppentherapie
}

\section{Pierre Loeb}

Past-Präsident Schweizerische Akademie für Psychosomatische und Psychosoziale Medizin SAPPM

\footnotetext{
* Zur besseren Lesbarkeit wird die männliche Form verwendet - gemeint sind immer sowohl Ärztinnen wie Ärzte, Inhaber wie Inhaberinnen, Patienten wie Patientinnen usw.
}

Korrespondenz:

Dr. med. Pierre Loeb

Winkelriedplatz 4

Postfach

CH-4008 Basel

Tel. 0613611020

Fax 0613612972

loeb[at]sunrise.ch

\section{Vorgeschichte}

1999 wurde von der Ärztekammer der FMH auf Antrag der damaligen Kommission Weiter- und Fortbildung (KWFB) ein spezieller Fähigkeitsausweis für psychosomatische und psychosoziale Medizin (FA PPM) verabschiedet, der am 1.1.2000 in Kraft trat. Mit der Revision TARMED 1.6 vom 1. 4. 2009 wurde die neue Tarifposition 00.0525 Psychosomatische Therapie (Einzelsetting), pro 5 Min. für die Inhaber* des Fähigkeitsausweises der Schweizerischen Akademie für Psychosomatische und Psychosoziale Medizin SAPPM eingeführt.

Der schon 1999 gleichzeitig eingereichte Antrag für eine Gruppentherapie-Position wurde damals zurückgezogen, da wegen zeitraubender Detaildiskussionen die Erteilung der Position für Einzeltherapie sonst zurückgestellt worden wäre. Gruppentherapie ist als Methode jedoch wichtig, effizient und auch kostengünstig. Dies hat uns veranlasst, den Antrag im April 2011 neu zu formulieren, und diesmal wurde ihm stattgegeben, und mit der Tarifversion 1.09 per 1.4.2013 wird die neue Psychosomatische TARMEDTarifposition 00.0526 «Psychosomatische Therapie (Gruppensetting), pro 5 Min.» in Kraft treten.

\section{Begründung für psychosomatische Therapie}

Die grundsätzlichen Gedanken für die Schaffung eigener Positionen für die Inhaber des Fähigkeitsausweises SAPPM (Schweizerische Akademie für Psychosomatische und Psychosoziale Medizin) wurden mit der Schaffung der Tarifposition 00.0525 durch die Tarifpartner anerkannt. Die spezielle Begründung für die Gruppentherapie-Tarifposition und weshalb sich diese zusätzliche Tarifposition von der bestehenden Gruppenpsychotherapie-Position der Fachärzte für Psychiatrie und Psychotherapie unterscheidet, wollen wir hier erläutern. Schliesslich geben wir auch unserem Bedauern Ausdruck, dass der Mut für grosszügigere, weitsichtigere Lösungen ausblieb.

Träger des FA PPM benötigen eigene TARMEDTarifpositionen mit denen sie ihre speziell erworbene Kompetenz abrechnen und auch statistisch vergleichen lassen können (Datenpool santésuisse und Trust Centers). Ältere psychosomatisch tätige Kollegen bedienen sich dazu der psychiatrischen Positionen, die sie dank ihres vertraglich zugesicherten Besitzstandes verwenden können. Jungen Kollegen, die den FA PPM seit weniger als 3 Jahren vor TARMED-Einführung erworben haben, steht diese Möglichkeit nicht zur Verfügung, und es entgeht ihnen damit die Möglichkeit, ihre besondere Kompetenz adäquat und transparent abzurechnen. Anders als in anderen Ländern Europas, wo es eine Abrechnungsziffer für psychosomatische Therapie gibt, gewährt TARMED keine adäquate finanzielle Entschädigung: Der Psychosomatiker erhält pro 5 Minuten gleich viel wie jeder andere Hausarzt, der z.B. mit der Position 00.0520 abrechnet. Dies ist schon deshalb unfair, weil der Psychosomatiker für den Erwerb seines Fähigkeitsausweises eine berufsbegleitende 2-jährige Weiterbildung absolvieren muss, die während seiner ganzen beruflichen Laufbahn durch eine Supervision begleitet werden muss, die auch für die Rezertifizierung verlangt wird. Diese Weiterbildung und lebenslange Supervision sind sowohl zeitaufwendig als auch teuer. Noch stärker auf den Bruttolohn wirkt sich aus, dass die oft schwierige und sozial unterschichtige Klientel kurzfristig Termine absagt oder gar nicht zur Sitzung erscheint und dann lange Termine unbezahlt bleiben. Schliesslich kann der Psychosomatiker seine Infrastruktur wie Labor, EKG und andere technische Leistungen nur zu einem kleinen Teil amortisieren.

\section{Den jüngeren Titelträgern des FA SAPPM stand keine adäquate Tarifposition für die Abrechnung zur Verfügung.}

Die TARMED-Kommission ist dieser Argumentation jedoch nicht gefolgt. Es wird Aufgabe des SAPPM-Vorstands sein, die zähen Verhandlungen fortzuführen, denn wir hoffen weiter, dass mit Anerkennung unserer wichtigen Arbeit und den Kosteneinsparungen, die wir dem Gesundheitswesen durch Reduzierung des Doktor-Shoppings und überflüssiger Abklärungen bringen, mittelfristig eine faire Anpassung erfolgen wird.

Der FA PPM wurde von der FMH als fächerübergreifender Fähigkeitsausweis geschaffen und muss allen Kollegen jeder medizinischen Fachrichtung bzw. Fachgesellschaft mit dem Zusatztitel FA SAPPM die Möglichkeit bieten, psychosomatische Therapien transparent und monitorisierbar abzurechnen. Die TARMED-Tarifposition 02.0050 Psychiatrische Diagnostik und Therapie, Gruppentherapie, pro 5 Min. ist jedoch explizit und abschliessend den Fachärzten Psychiatrie und Psychotherapie vorbe- 
halten. Die Abrechnung einer Gruppentherapie blieb somit nur den Titelträgern SAPPM, die noch über den Besitzstand Psychiatrie verfügen, vorbehalten. Den jüngeren Titelträgern des FA SAPPM stand somit keine adäquate Tarifposition zur Abrechnung zur Verfügung, um Gruppengespräche adäquat und transparent abzurechnen.

\section{Gruppentherapie-Angebote und Indikationen} Psychosomatische Therapieangebote im Gruppensetting haben sich bei Patienten aus dem Kreis psychosomatischer Störungen als effiziente und kostengünstige Therapiemethoden erwiesen, die der Isolation und dem Rückzug der Patienten entgegenwirken und eine aktive Verarbeitung und Anpassung unterstützen. Vor allem die Vorbild- und Unterstützungswirkung durch andere, an ähnlichen Störungen leidende Gruppenmitglieder hat sich als sehr hilfreich und motivierend erwiesen.

$\mathrm{Zu}$ den Therapieverfahren, die für den Erwerb des Fähigkeitsausweises vorausgesetzt werden, gehö-

\section{Rahmenbedingungen für die psycho- somatische Gruppentherapie}

Leider wurden unsere Vorschläge, zwischen zwei Gruppengrössen zu unterscheiden, von der Kommission erneut nicht übernommen, und es bleibt für die neue psychosomatische Gruppentherapie bei denselben Rahmenbedingungen, wie sie von der Psychiatrischen Gruppenpsychotherapie 02.0050 vorgegeben sind. Es gibt nur die eine Gruppendefinition, von 3 bis 10 Personen, die Behandlung durch bis zwei Gruppenleiter und die maximale Sitzungsdauer von 105 Minuten, was für grössere Gruppen viel zu kurz bemessen ist (wenn jedes Mitglied sich ein- oder zweimal zu Wort meldet, etwas Neues erlernt und eingeübt werden soll, was ja die Idee einer solchen Sitzung ist). Einzig die Co-Therapie durch einen Facharzt oder einen delegiert arbeitenden Psychotherapeuten ist neu möglich.

Schliesslich soll auch noch die Divisormethode kritisch hinterfragt werden. Abrechnung nach Divisormethode bedeutet, dass die Gesamtzeit tariflich auf

\section{Von keiner anderen ärztlichen Gruppe wird verlangt, dass ihr Idealismus und ihre Kompetenz nicht fair und betriebswirtschaftlich korrekt abgegolten wird.}

ren auch Entspannungsverfahren, Familien- und Gruppengespräche sowie weitere Therapieangebote im Gruppensetting (vgl. Psychosomatische und Psychosoziale Medizin SAPPM, Fähigkeitsprogramm vom 1.1.2000, Fertigkeiten, Kapitel 4.2.2, S.4).

Psychosomatische Therapieverfahren bestehen oft in Psychoedukation, ein Verfahren, wozu sich das Gruppensetting besonders eignet, indem es Zeit einspart (mehrere Personen werden gleichzeitig unterrichtet) und durch die Gruppenerfahrung (Wettbewerb, Rivalität, Gruppenreiz) auch nachhaltiger wirkt. Therapieangebote im Gruppensetting sind in der Literatur als besonders effizient belegt [1]. In der Regel wird dies durch die erleichterte Erreichung von Therapiezielen begründet, da die Teilnehmer voneinander besser lernen. Gerade bei Störungsbildern, in denen das ängstliche Vermeiden bestimmter Situationen oder Verhaltensweisen eine wesentliche Rolle spielt, ist das Lernen am Modell des Mit-Patienten ein wichtiger Wirkfaktor der therapeutischen Intervention.

Dies zeigt auch eine Rückfrage im Rahmen einer Abklärung der Leistungspflicht durch die ProvitaGesundheitsversicherung vom 1. Februar 2012: Unter Punkt 9 wird gefragt: «Handelt es sich um eine Gruppentherapie? Wenn nein, weswegen wird keine solche durchgeführt?» Offensichtlich besteht ein grösseres Interesse der Kassen an dieser Behandlungsmethode. die anwesenden Patienten verteilt werden muss. Dauert eine Sitzung $10 \times 5$ Minuten, und es sind 10 Patienten anwesend, bezahlt jeder Patient $10 \times 5 / 10=5$ Anteile. Fehlen jedoch 2 Patienten, bezahlen die 8 anwesenden Patienten $10 \times 5 / 8=6,25$ Anteile. Dass das Wegbleiben von einer Gruppensitzung den andern Teilnehmern aufgebürdet wird, macht edukativ gesehen keinen Sinn.

\section{Fazit}

Die Schaffung der neuen Tarifposition freut uns sehr. Dennoch bedauern wir, dass konstruktive Vorschläge für Besserungen und echte Neuerungen nicht aufgenommen wurden. Schliesslich muss die inadäquate Honorierung einer besonders erlernten Kompetenz angeprangert werden. Psychosomatiker sind Idealisten - doch von keiner anderen ärztlichen Gruppe wird verlangt, dass ihr Idealismus und ihre fachlich erworbene Kompetenz nicht fair und betriebswirtschaftlich korrekt abgegolten wird.

\section{Literatur}

1 Petermann F. Patientenschulung und Patientenberatung - Ein Lehrbuch. Göttingen: Hogrefe-Verlag; 1997. Alsleben H. Thieme Verlag PiD 4 -2005. 6. Jahrgang, www.thieme.de/pid/leserbriefe/pdf/news1.pdf

Pitschel-Walz G, Bäuml J, Kissling W. Psychoedukation Depression - Manual zur Leitung von Patienten- und Angehörigengruppen. München/Jena: Urban \& Fischer Verlag; 2003.

Strauß B, Dankwart M. Gruppenpsychotherapie: Lehrbuch für die Praxis. Heidelberg: Springer; 2012. 